\title{
La Crise de l'administration de la Justice Criminelle.
}

\section{Manoel Pedro Pimentel}

Professeur titulaire de la chaire de Droit Pénal de la Faculté de Droit de l'Université de São Paulo et Juge du Tribunal de Alçada de l'Etat de São Paulo.

Sous le patronage de l'Institut des Avocats Brésiliens, siégé à l'Etat de la Guanabara, a eu lieu un congrès d'études dont le thème a été $L a$ Crise de l'administracion de la Justice Criminelle, à Rio de Janeiro, du 30 au 31 de octobre 1972 .

Sous la présidence du Dr. Theophilo de Azeredo Santos et sous la coordination du professeur Virgilio Luiz DonNicI se sont réunis de nombreux spécialistes, des professeurs univesitaires, des magistrats, des membres du Ministère Public, des avocats, des Préfets de Police de plusieurs Etats brésiliens, pour discuter des problèmes de la plus grande actualité à l'administration de la Justice Criminelle.

Trois thèmes ont été distribués aux groupes de travail:

Groupe I - La criminalité traditionelle;

Groupe II - Les Crimes sans victimes; et

Groupe III - Le coût du crime et la prévision de la criminalité.

La matière examinée par le Groupe I, dont on nous a désigné rappor. teur, avait pour but l'examen de la criminalité traditionnele considérée sous de divers points de vue selon une table de matières préalablemen. organisée, savoir:

I - Caractéristiques de la société moderne;

II - Réaction sociale contre la criminalité; 
III - Evaluation du crime par rapport au système policier;

IV - Evaluation du crime par rapport au système judiciaire; finalement,

$\mathrm{V}$ - Evaluation du crime par rapport au système pénitentiaire et,

VI - Son examen par rapport aux homicides et aux crimes contre le patrimoine.

\section{CONSIDÉRATIONS PRELIMINAIRES.}

La position prise par l'Ecole Technico-juridique, en écartant du Droit pénal scientifique toutes les questions étrangères à la dogmatique, a déterminé un éloignement progressif de la Criminologie, dont le contenu est enrichi par les sciences sociales, par la médecine, par la philosophie, par l'economie; il résulte un vidage de la science pénale, astreinte à l'étude des normes de droit positif.

La conséquence la plus évidente de ce progressif éloignement a été la diminution de l'importance de l'inclusion de la criminologie comme objet d'études des Facultés de Droit. Abandonnée par les juristes, la Criminologie a trouvé son gite dans d'autres secteurs de la connaissance, spécialement dans le champ de la Médecine Légale de laquelle elle n'est devenue qu'un simple segment. Une conséquence forcée qui découle de ce nouveau schéma a été le manque de préparation des juristes à l'égard des forces vives qui - que les purs dogmatistes le veuillent ou non continuent à instruire le Droit pénal.

Le crime est un phenomène social, ayant ses racines dans la réalité, dont la connaissance dépend de l' analyse de plusieurs facteurs sûrement étrangers à la dogmatique pénale. Cependant, la loi pénale décrit, dans son précepte primaire, une conduite concréte sortie de cette réalité même et considéré comme offensive à biens et intérêts qui doivent être protégés juridiquement.

Cette interaction est trop éloquente, elle s'impose à n'importe quel observateur. Le législateur pénal puise au des études criminologiques pour déterminer les préceptes primaires des lois en leur imposant les sanctions respectives. En outre, la connaissance des causes du crime aide à l'élaboration des plans de prévention du délit qui pésent aussi sur la pragmatique des types pénaux de péril. 
La vérité est que les changements sociaux, les nouvelles découvertes scientifiques, les progres techonogiques et les facteurs économiques favorisent l'apparition de conduites offensives inusitées qui ne sont que tardivement apperçues par le juriste pur. La criminologie par ses caractéristiques éclectiques, est une source de la plus grande valeur pour le Droit pénal. Et, il faut en convenir, elle ne peut plus rester à l'écart des études juridiques.

Un Côde pénal exige un travail pénible d'élaboration législative. Le plus souvent, cependant, quand il est promulgué, il souffre un décalage, au moins en partie, par rapport aux faits sociaux. Les changements rapides auxquels nous avons fait allusion ci-dessus continuent en rythme vertigineux, tandis que les lois restent immobiles. Il y a une très compréhensive tendance à résister, d'une façon conservatrice, en ce qui concerne l'élaboration de nouvelles lois. La raison en a été démontrée à satiété par Eduardo Novoa Monreal: "Nous avons observé que le droit positif, aussi bien dans les systèmes de législation écrite, que dans ceux d'expérience coutumière, a une tendance naturelle à devenir stable. L'élabo ration difficile des formules législatives, en particulier celles codifiées, tout d'abord et la permanence, s'étendant à l'avenir, des usages et des coutumes précendentes, ensuit, exercent sur le droit positif une influence stratifiante qui tend à consolider des lois antiques et les projeter jusqu'à l'avenir, avec une pretention de validité plus ou moins durable. Cependant, les changements sociaux, avec leur surprenante mobilité outrepassent, tout à fait, les schémas juridiques et finissent par les laisser de côté comme une chose inutile troublant le progrès d'une humanité qui semble avoir atteint un des points culminants dans son effort vers la maitrise de la nature" 1 .

On y doit ajouter que, au Brésil, il y a plus de 80 ans, on ne discute pas l'élaboration d'un Côde pénal par l'initiative du Pouvoir législatif. Le Côde pénal de 1940 a été édité par un Décret-loi, número 2.848, du 7 décembre 1940. Et le Côde pénal de 1969, qui n'est pas encore en vigueur, l'a été par le Décret-loi n. ${ }^{\circ} 1.004$, du 21 octobre 1969. Par conséquent, pas même les débats, si nécessaires parmi les divers représen-

1. EDUARDO NOVOA MONREAL, Progrés Humain et Droit Penal, in Revista de Direito Penal, n. 2, abr./jun., 1971. 
tants du peuple et apportant des opinions et des observations des classes professionnelles les plus variées, ont enrichi de leur contenu authentique les lois pénales destinées à régir la réalité brésilienne.

Par cela même et avec beaucoup de raison, Manuel Lopes ReY, en se référant au Côde Pénal de 1969 a remarqué que "le projet brésilien à l'appui des 116 articles de sa Partie Générale cite le projet allemand 16 fois, le Côde pénal de la Yougoslavie 12, le Côde grec 8, l'italien 6 fois, en se rapportant occasionellement à d'autres côdes. On n'y trouve aucune information sur la réalité nationalle" 2

On comprend aisément que la loi pénale, éloignée de la réalité existant aux lieux où elle doit être appliquée ne contribue pas à la réalisation da la justice criminele. De cette façon, on identifie un des principaux aspects de la crise où nos vivons. Les nouvelles exigences sociales, créeant des inédites de délit, ne sont pas dûment considérées dans le contexte des lois pénales qui ont besoin d'information précises.

\section{CARACTÉRISTIQUES DE LA SOCIÉTÉ MODERNE.}

La transformation fantastique de la société pendant les derniers 70 ans a placé des problèmes terrifiants. Jamais l'homme ne s'est trouvé en présence de changements si nombreux et si profonds comme pendant ce siècle. Le progrès technologique a bouleversé de fond en comble la face de la terre, en amondrissant le monde par la rapidité des communications. La machine a dominé l'homme qui s'étonne de la précision des machines électroniques se pâmant d'admiration devant sa créature, capable de le subjuguer. L'industrialisation a causé, en conséquence, le besoin de marchés toujours renouvelés pour consommer la production toujours croissante. D'où le prestige de la propagande, explorant les masses, créeant de désirs artificiels, dirigeant tout, dans un véritable processus de destruction.

En souffrant une telle pression, en présence de si nombreuses charges et sollicitations, il semble que l'homme s'aliène. Et, ce qui est plus

2. MANUEL LOPES REY, Quelques considerations analytiques sur la criminologie et la justice criminalle, in Revista de Direito Penal, n: ${ }^{0}$, out./dez., 1971. 
troublant, le processus, semble-t-il, n'offre pas d'option. Revenir en arriére ce serait reculer et peu de personnes en seraient d'accord; pourtant à leur avis ce serait la seule solution sûre.

A ce propos, MANUEL LOPES REY a signalé: "l'industrialisation, l'urbanisation et les changements sociaux sont généralement indiqués comme des explications qui embrassent toute l'extension et gravité du crime. Son caractére général et vague signifie plutôt l'admission de l' échec de la criminologie contemporaine qu'une sûre affirmation criminologique. Assurément l'industrialisation et l'urbanisation, ou peut-être la forme par laquelle elles sont généralement réalisées, provoquent de nouvelles formes de crime et en aggravent d'autres; cependant, si l'on tient compte de leur compléxité sociale, politique, économique et culturelle, et jusqu'à un certain degré l'évolution qu'ils représentent est nécessaire, voire même inévitable, les offrir comme des explications causales du crime, montre l'impasse où la criminologie contemporaine est arrivée" 3 .

En effet, ce serait une simple confession de faute impotente, qui serait automatiquement justifiée, qu'admettre que le progrès technologique et l'industrialisation, à côté de l'urbanisation, ne se font que pour le bien de l'humanité, bien qu'aux frais de la vie, de la santé, du bien-être et du bonheur de millions d'hommes. Il faut plus qu'une attitude de résignation parce que la dignité de la personne humaine - ne fút-ce que d'une seule personne - doit toujours être considérée comme quelque chose à être respectée.

Si l'on aurait su que, n'importe où, une épidemie aurait tué, en une année, des milliers de personnes, les organismes chargés de la santé publique tàcheraient de donner une solution immédiate à ce problème. Toutes les nations contribueraient, solidairement, en envoyant des ressources médicales, des vaccins, de la nourriture, tout ce qui serait nécessaire pour vaincre la cause de la mortalité en masse.

Très bien, à São Paulo, selon un rapport officiel signé par $\mathrm{Mr}$. le Directeur de la DET-1, l'ingénieur IsAo KonNo, la voiture automobile a tué 1883 personnes en 1970, et 2386 personnes en 1971. Au Brésil, d'après les renseignements donnés par l'ingénieur BARUCH MEIER GRINI.AT, à peu près 10 mille personnes sont mortes en 1971 et 200 mille ont été blessées dans la même période. On affirme qu'aux Etats Unis, pendant la

3. MANUEL LOPES REY, art. cit., p. 23. 
Deuxième Guerre Mondiale, 947 mille personnes ont été tuées aux champs de bataille tandis que les accidents routiers ont tué 3,3 millions de personnes pendant la même période, en dépit du rationnement du combustible.

Il n'y a pas de doute que le progrès est devenu un dévorateur d'hommes, comme Saturne qui consumait ses propres enfants. Nous vivons dans une jungle urbaine et nous nous sentons ménacés. Les effets de cette ménace sont les états d'angoisse, de dépression éveillant l'agressivité; comme le Docteur JaIr PINHeIRo QueIRoz a dit au Congrès Brésilien de Réflexologie qui a eu lieu tout récemment à São Paulo: "La neurose urbaine a été constatée en des proportions chaque fois plus grandes dans les grands rassemblements urbains, sous la forme des maladies mentales les plus diverses. C'est la forme la plus récente par laquelle l'industrialisation attaque l'être humain. Les grandes centres urbains conditionnent l'apparition de conduites neurotiques, qui apparaissent à travers les réactions qui s'enchainent sucessivement, jusqu'à ce qu'elles atteignent leurs dernières extremités. L'excès d'agressivité, l'angoisse, la difficulté de concentration, la tristesse, l'accablement sont quelques élémests communs à la neurose urbaine. On meurt peu de folie, mais l'accroissement accéléré de son porcentage parmi les maladies conduisant à l'infirmité a atteint un poit qui cause dejà un certain alarme".

Dans ce même ordre d'idées, il a rappelé qu' "une des conséquences des neuroses est la massification, où l'individu voit son individualité dévorée par les masses. La valeur d'un évènement est mésurée par le nombre de personnes présentes et non pas par le niveau des personnes présentes. Ce type de considérations peut porter l'homme à éprouver un sentiment de manque d'amour pour se vie et pour celle des autres personnes, parce qu'il n'est pas conditionné à donner de l'importance à une seule mort. L'homme peut aussi se sentir sans valeur quand il est isolé d'un groupe grand"

Le besoin de sûreté conduit l'homme à préférer l'abdication de sa liberté pour se dissoudre dans un groupe puissant en se faisant illusion d'une sensation de force. Des phénomènes comme celui-ci ont favorisé la triste réalité ou le fascisme et le nazisme se sont consitués; il ne faut pas rappeler leurs crimes.

A la racine de la violence croissante constatée dans les formes de délit qui se multiplient dans la société moderne, se trouve certainement l'influence de ces facteurs. Pressionné par tous côtés, l'homme réagi 
agressivement. Les moyens d'information, le cinéma, la télévision, la radio, la presse, contribuent, à leur façon, à la divulgation des nouvelles méthodes d'action. Nous assistons ébahis à une réaction en chaine, car il y a une marée montant d'homicides, d'assauts aux banques, aux établissements particuliers, aux chaufeurs, pour ne pas parles des séquestrations et des actes de vandalisme.

Le crime organisé par des groupes idéologiques, ayant des buts po. litiques, sert comme exemple aux criminels et est tout de suite imité. Les séquestrations d'avions ont motivé, sur, une échelle plus petite, les séquestrations d'autobus urbains, la forme la plus récente de crime qui vient d'apparaitre à São Paulo .

\section{LA REATION SOCIALE CONTRE LA CRIMINALITÉ.}

On observe qu'il existe actuellement dans les pays développés une louable préoccupation pour le problème de la criminalité.

Aux Etats-Unis elle est signalée parce qu'on trouve aux plates-formes électorales des candidats à la présidence des affirmations expresses sur le combat au crime. Dans la pratique, plusieurs Comissions ont été constituées, en niveau officiel, pour étudier la criminalité. Qu'on ne mentione, pour amour à la brièvetè, que la LeaA (Law Enforcement Assistance Administration) qui a reçu des ressources budgétaires substantielles: 63 millions de dollars en 1969; 268 millions en 1970; 530 millions em 1971; 669 millions en 1972 et 850 millions de dollars en 1973 .

$\mathrm{Au}$ Canada, on met en évidence la Commission Prevost (1970) sur l'Administration de la Justice Criminelle dans Providence de Québec. L'Angleterre, avec le Criminal Justice Act (1948), agit à travers le Home Office Research Unit, en cherchant les causes de la criminalité et en s'occupant du soin des criminels.

Au Brésil, malheureusement, il n'y a pas d'organisme officiel semblable. De petites contributions sont donnés par des organisations publiques et l'on peut trouver quelques donnés statistiques. Il n'y a cependant pas une centralisation des données pour des études dirigées et recherches d'information. A São Paulo, tout récemment, le Gouvernement de l'Etat a décidé de développer la politique pénale et pénitentiare, en four- 
nissant des moyens à l' Institut "Oscar Freire" et en le chargeant de son execution. Cepedant, à l'exception de la réalisation d'un cours, nous ne savons rien sur son activité, notamment sur des recherches pratiques.

D'un autre côté, il y a un divorce presque total entre le Pouvoir Judiciaire, la Police et l'Université. La criminologie par son caractère éclectique devrait être le trait d'union unissant tous les efforts pour planifier convenablement le combat au crime. Nous ne voyons cependent pas une ferme tendance vers ce sens-là .

La législation de combat aux toxiques, par exemple, est improvisée, ne faisant pas face à la profondeur du problème, parce qu'elle ne comprend pas tous ses aspects à cause du manque d'informations et de données complètes.

L'indifférance ou l'ignorance de la véritable extension du problème de la criminalité a fait São Paulo perdre sa condition de siège de l'Institut Latin Américain de Criminologie, organe de l'ONU, qui a été installé au Brésil après une pénible dispute avec d'autres pays qui y aspiraient. L'insensibilité des organes officiels et des responsables par le secteur a touché les limite de l'incroyable.

Nous pouvouns donc conclure qu'au Brésil la contribution officielle pour le contrôle par rapport aux nouvelles formes de criminalité est minime.

III. EVALUATION DU CRIME PAR RAPPORT AU SYSTÉME POLICIER.

Une vision réelle du système policier démontre que nous ne trouvons pas encore en condition de faire face à la marée montante de criminalité.

Cependant, du point de vue de la police répressive il y a du progrès. Les organes policiers se sont bénéficiés de plus grandes resources techniques et de personnel. Des moyens de comunication plus efficaces, des voitures, du matériel de travail ont contribué à rendre les services poliliciers, dont la structure est en train d'être remodelée et actualisée, plus dynamique.

Le plus grande problème est encore celui du recrutement et de l'entrainement du personnel des échelons inférieurs. Tandis que, en quelques 
Etats du Brésil, les préfets de police reçoivent une formation universitaire, en suivant des cours juridiques et, postérieurement, des cours de perfectionnement à l'Ecole Supérieure de Police, ce n'est pas encore possible d'obtenir la même formation aux dégrés initiels de la carrière. Les basses rétributions, les risques professionnels, l'exigence d'un horaire de travail très pénible, écartent les candidats les mieux doués qui cherchent ailleurs une plus grande satisfaction de leurs besoins.

Pourtant, il faut le reconnaitre, il existe la préoccupation officielle d'améliorer les organismes policiers, quoiqu'une plus grande importance soit placée sur le système répressif. Voilá pourquoi le pourcentage des délits qui pourraient être évités moyennant une prévention planifiée et rationnelle, est encore élevé. Par conséquent le chiffre noir est aussi élevé. Plusieurs infractions ne sont pas convenablement recherchées et d'autres, à cause de leur petite importance, sont tout simplement transcrites sur les registres policiers.

Les chiffres figurant dans les statistiques démontrent que les crimes plus nombreux sont tout d'abord ceux de blessures commises avec dol ou par négligence ou par imprudence, ensuite le vol. Ces deux classes d'infractions représentant $50 \%$ du total des crimes commis. Dans une enquête que nous avons fait faire, menée à bout par Bibliothèque du Tribunal de Alçada en 1965, nous avons conclui que les délits de blessures commises avec dol, objet de notre étude, représentaient, parmi 551 prévenus, 447 illettrés et 81 ayant une instruction primaire; les criminels dont l'âge variait entre 20 et 30 ans représentaient le pourcentage le plus élevé, soit 269 prévenus.

Par rapport aux blessures constituant des délits par négligence ou par imprudence leur nombre a été 441 dont $40 \%$ a eu lieu à São Paulo et plus fréquemment pendant l'été. En ce qui concerne l'âge des infracteurs, on a remarqué que ceux entre 25 et 35 ans étaient les plus nombreux. Comme la loi empêche les illettrés d'obtenir leur permis de conduire, on n'a observé que 12 cas d'absence d' une telle instruction.

Les crimes de vol ont été plus nombreux dans la Capitale, leur pourcentage représentant plus de $50 \%$ des cas, et ils ont été pratiqués pendant n'importe quelle époque de l'année. Les infracteurs dont l'âge se trouvait entre 18 et 25 ans étaient les plus nombreux. Les infracteurs illéttrés ou ayant une instruction primaire représentaient presque $97 \%$ du total. 
Ce petit tableau donne une idée de l'immense travail auquel la police doit repondre, surtout dans les villes d'une plus grande concentration urbaine. On y doit ajouter que, par l'imposition de la loi $n .^{\circ}$ $4.611 / 65$, l'instruction contradictoire des crimes d'homicide et de blessures non intentionnelles doit être réalisée par l'autorité policière, ce qui rend son travail plus difficile.

Mallheureusement, on constate que la règle de la présomption d'innocence ne s'est pas encore imposée dans les milieux policiers. Le suspect continue à être suspect jusqu'à ce qu'il fournisse une preuve contraire. L'accusation présentée contre l'inculpé le rend presumablement inculpé et il est traité comme tel pendant toute la durée de l'enquête policière.

Très rarement l'inculpé est traité comme un innocent et cependant celle-ci devrait être la conduite normale. La question reste ainsi un mal traditionnel.

IV

EVALUATION DE LA CRISE PAR RAPPORT OU SYSTËME JUDICIAIRE.

La justice criminelle au Brésil n'est pas encore suffisamment douée de moyens pour atteindre ses vrais buts. Il subsiste encore l'idée d'admiministration de la justice, nom qui donne une idée très claire du caractère bureaucratique des services judiciaires. Ce concept a une force d'actualité, parce que le propre Côde pénal de 1969, dans son chapitre III, au Titre XI continue à donner aux infractions y prévues le nom de crimes contre l'administration de la justice.

Administrer la justice n'est qu'une partie de la charge totale de la réalisation de la justice. Attachée à cette idée partielle, la justice criminelle est passive, statique, lieu d'être active, dynamique. On peut montrer quelquer exceptions qui servent, tout d'abord, à confirmer la règle et, ensuite, à démontrer qu'une réalisation dynamique de la justice criminelle est possible pour aboutir à solution courageuses pour les problèmes qui continuent à nous défier. Le Tribunal de Justice de l'État de São Paulo a donné un exemple de ce dynamisme salutaire, quand, à travers des ordonnances du Conseil Supérieur de la Magistrature, il a institué la prision auberge et la prison domiciliaire, en dévançant le législateur ordinaire en ce qui concerne la politique pénitentiaire. Son action a été encore plus 
remarquable, quand, par l'intermédiaire de son Président, Mr. le Desembargador Cantidiano de Almeida, il a dénoncé les activités du "Esquadrão da Morte" * pour investiguer son action et établir ses responsabilités. Ces deux exemples nous font sentir la présence de cette action dynamique qui favorise une véritable réalisation de la justice criminelle et non pas simplement son administration passive.

Presque tous les champs de l'activité humaine ont recueilli les résultats $\mathrm{du}$ progrès technologique et en ont profité. La justice criminelle n'y est pas incluse. Seulement la machine à écrire, les appareils enrégistreurs et amplificateurs de son y ont été reçus et ces derniers d'une façon très restrictive.

Le Tribunal de Alçada criminelle de São Paulo est passé outre, parce qu'il a adopté des systèmes de microfilmage de documents et procèsverbaux pour ne pas entretenir des archives très volumineuses. On y est en train do monter un centre de cybernétique pour consulter les décisions doctrinales et jurisprudentielles. Quelques sections du pouvoir judiciaire se servent du telex, comme moyen de communication, mais le petit nombre d'appareils installés en limite l'emploi.

Les formules de procédure enracinées, imposant l'enregistrement par écrit de tous les actes judiciaires, conserve la tradition de former des procès-verbaux contenant des pièces non nécessaires. Les actes d'instruction n'ont pas souffert de changements remarquables pendant les dernières 50 années. On n'a pas encore songé à donner aux compagnies spécialisées en cybernétique le soin de trouver de nouvelles solutions pour permettre une révolution dans les méthodes employées jusqu'ici.

Il n'y a pas de recherches ayant pour but d'abréger, sans risquer l'administration de la justice, les actes de procédure. Les machines électroniques ne sont pas encore entrées dans les Tribunaux pour y être expérimentées. Il y a une grande distance entre la routine des travaux judiciaires et la technologie moderne.

Par cela même, l'accumulation des services est la règle générale. L'accroissement permanent de la population exige une augmentation correspondante de l'appareil judiciaire. On y pare en augmentant le nombre des juges et des sièges aux Tribunaux. Pour y donner une idée, on constate qu'à l'Etat de São Paulo, en 1962, il y avait 9 magistrats au Tribu-

* Dénomination populaire de certain organisme policier qui-dit on-execute sommairement les criminels. 
nal de Justice et 9 magistrats au Tribunal de Alçada pour juger les appelations criminelles. En 1972, il y a au Tribunal de Justice le même nombre de magistrats mais le Tribunal de Alçada s'est démembré en deux Tribunaux de Alçada civile et un Tribunal de Alçada criminel, ce dernier ayant 24 juges en travail de jugement. Donc, em dix ans le nombre des juges pour les appelations criminelles est presque trois fois plus grand au Tribunal de Alçada criminel. Quand le Tribunal de Alçada de l'Etat de São Paulo a été créé, en 1951, en estimait excessive la quantité de travail des juges: 312 procès par an. Aujourd' hui, en moyenne, chaque juge du Tribunal de Alçada criminel le doit proférer à peu près 750 votes par an, deu fois de plus la quantité déjà considérée insuportable à l'époque mentionnée.

Le coût de ces services est très élevé, car les projets de loi 216 et 218, publiés aux éditions du "Diário Oficial", le 7 et le 10 octobre 1972, ont consigné une somme de Cr\$11.169.588,00 au Tribunal de Alçada, tandis que l'ensemble des services du Tribunal de Justice a reçu pour ses dépenses $\operatorname{Cr} \$ 231.880 .747,00$.

A côté de cette véritable stagnation de l'appareil judiciaire, on constate que la justice criminelle souffre d'autres maux. Tout d'abord, il n'y a pas une justice criminologique. La jurisprudence continue à être la même casuistique d'il y a 30 ans, sans aucune ouverture aux solutions qui présentent une nouvelle vision des problèmes sociologiques modernes. Fixés à des principes étanches, bloqués par la dogmatique, les magistrats tendent à la répétion des conceps et aux interprétations sans validité actuelle ou sans correspondence effetive à la réalité dont la mutation, nous l'avons dejà vu, se fait à l'insu de a loi pénale outrepassée.

Il y a sans doute une croyance enracinée selon laquelle les juges criminels n'ont pas besoin d'être les plus cultivés ni les mieux doués. Ces dons sont réservés à la juridiction civile, où, on le croit, il $\mathrm{y}$ a plus de difficultés. Dejà en premier degré de juridiction on perçoit cette tendance. Il semble que les juridictions criminelles n'ont pas besoin de juges ayant beaucoup d'aptitudes, on y destine les magistrats les plus inexpérimentés.

Comme conséquence de cet état de choses, il est en train de se former, parmi les juges, une certaine prévention contre la judicature criminelle à tel point qu' on n'y laisse pas rester les magistrats désireux de briller et d'obtenir du succès dans la carrière. 
On n'en comprend pas très clairement la raison.

Les valeurs confiées aux criminels sont égales voire même supérieures à celles confiées aux juges civils. Cependant celle-là n'est pas la réalité constatée.

Un autre aspect remarquable, qui favorise la crise, est le manque de spécialisation des juges criminels. Très souvent réclamée par les spécialistes, elle n'a jamais été l'objet des préoccupations du législateur. Si l'on additionne ce manque de spécialisation et de connaissances criminologiques suffisantes à l'absence d'informations correctes au sujet de la personalité de l'accusé, l'individualisation de la peine est un mythe.

Une telle situation explique la persistence d'une tradition répressive, alors même que les modernes perspectives pénales et criminologiques montrent d'autres solutions découvertes grâce à un incoditionnement de la personalité du juriste pénal, bénéficié par les apports de la criminologie.

On y doit encore ajouter l'inefficacité des peines de prison, dominantes dans notre système punitif, pour avoir un tableau final de la crise, dans lequel le nombre des récidives est effrayant, témoignant les erreurs de tout le sistème et l'inutilité de si grandes dépenses qui ne se justifient que par le besoin de répression du délit et ayant comme but l'écartement du délinquant du milieu social.

Ce tableu objectif nous montre la justice criminelle sous son véritable semblant. Et nous ne tomberions pas en erreur en affirmant qu'il est aussi un des motifs de la crise où elle se débat.

\section{EVALUATION DU CRIME PAR RAPPORT AU SYSTËME PENITENTIAIRE.}

La situation du système pénitentiaire est très délicate. $\mathrm{La}$ peine d'emprisonnement est devenue la forme principale de punition, à partir du dernier siècle, en remplaçant les travaux forcés, les châtiments corporels et la déportation. Cepedant, pas même l'arrivée de la science pénitentiaire a réussi à éliminer les graves inconvénients de ce type de peine qui est tombé en désuétude.

Le but utilitaire de la peine, qui tourne autor de la préoccupation de réhabilitation du délinquant, n’a pas été atteint dans le domaine de 
la prison. Le phénomène est reconnu même aux pays développés, où il y a des établissements d'emprisonnement exemplaires et où, malgré cela, on n'a pas obtenu des résultats satisfaisants.

Des tecniques thérapeutiques sont expérimentées témoignant qu'il est très difficile de conditionner convenablement, dans la prison, l'homme qui se prépare pour vivre en liberté. Des théories sont crées et ensuite abandonnées à cause de l'insuccès qui les accompagne dans la pratique. Le nombre des récidivistes démonstre la faiblesse des procédés de rééducation et de réhabilitation sociale. En vérité, l'accroissement de la criminalité exige un acroissement proportionnel de places dans les pénitenciers en inquétant les autorités qui en sont responsables, surtout parce que leur construction et leur maitien deviennent chaque fois plus coûteux.

A l'Etat de São Paulo, par exemple, pendant les dix dernières années on n'en construit qu'un seul, à la ville de Avaré.

Il a coûté au Trésor la somme de 16 millions de cruzeiros. Sa capacité est de 450 condamnés. Pour le maintenir l'Etat débourse un million de cruzeiros par mois. Très bien, d'après des données recueillies en sources officielles, il y avait, exactement, 12.000 condamnés recueillis dans les pénitentiers, tandis que leur capacité n'allait pas au-delà de 7.000 condamnés au maximum. Il y avait donc un excès de 5.000 prisionniers. Et pourtant à cette époque-là il y avait 41.965 mandats d'arrêt.

En supposant que ce nombre ait atteint, en trois ans, 50.000 mandats, d'après une information officieuse, on aurait besoin de 100 pénitenciers semblables à celui de Avaré. Sans tenir compte du temps que leur construction exigérait, on aurait besoin d'un deboursement de 1,6 billions de cruzeiros, dépense qui ne serait certainement pas considérée par le plus optimiste des budgets. Il suffit qu'on dise que, pour l'exercice financier de 1973, les oeuvres du Département des Instituts Pénaux de l'Etat de São Paulo atteignent la somme très modeste de 20 millions de cruzeiros .

Nous nous plaçons devant un problème apparemment insoluble ou, du moins, qui ne peut pas être résolu à travers les ressources conventionnelles. On doit trouver une nouvelle philosophie de la peine, en laissant de côté l'idée d'emprisonnement. À ce sujet, il serait utile de penser à un classement des criminels d'après un traitement à être appliqué au-dedans et au-dehors des prisons. Les établissements de la plus haute sûreté recevraient les criminels particulièrement dangereux. Les prisons de 
moyenne sûreté recevraient les délinquats moins dangereux en régime de demi-liberté. Les criminels qui ne représentant aucun danger à la société (les criminaloides) resteraient en liberté, dans un régime de prison auberge ou de prison domiciliaire.

Il conviendrait d'ériger les peines accessoires en peines principales, étant donné leur caractère immédiat, ce qui rend capables d'avoir un effet plus prononcé le condamné qu'une peine de détention suspendue conditionnellement.

On appliquerait un traitement convenable aux condamnés reccueillis dans prisons de la plus haute sûreté, d'après leur encadrement dans certains types.

Il serait convenable d'appliquer la technique employée para la psychologie expérimentale dont les résultats ont été très bons surtout quand elle à été appliqué à des groupes nombreaux d'individus. La méthode d'approches successives ou la technique du fading pourraient peut-être apporter les bénéfices atendus, étant appliquées à des condamnés dangereux et ayant pour but la modification profonde de leur personalité.

Les condamnés à des régimes de prison auberge ou prison domiciliaire seraient surveillés et appuyés par un personnel spécialement exercé qui aurait pour tâche de les faire rentrer en société sans d'autres charges pour l'Etat.

Les autres meneraient une vie normale, soumis à de petites restrictions, mais surveillés par des organes compétents.

Voilà, d'une façon générale, les suggstions pour l'agenda d'une réforme du système des peines si réclamée dans l'actualité.

Il vaut la peine de mentionner quelques manifestations officielles des autorités brésiliennes déclarant l'échec du présent système de peines. Sous le titre de "Motion de Pelotas", les membres du IV Congrès NAtional des Prefets de Police du Brésil, qui a eu lieu à Pelotas, dans l'Etat du Rio Grande do Sul, ont affirmé au § 4: “La gravité du problème des prisions au Brésil est telle que les pénitenciers régionaux qui seront construits sur le territoire national, ne recevront que l'excès des condamnés. On suggère, donc, une nouvelle philosophie de la peine de façon que les prisons de sûreté maximum soient destinés exclusivement aux condamnés présentant un grand danger et dont l'écartement devient indispensable à la tranquilité de la société". 
Cette motion est nu 23 juillet 1972. Antérieurement, le 7 juillet de cette même année, le IV Congrès du Ministère PUblic de Rio de JANEIRo approuvait la conclusion où l'on consignait que: "si le Côde pénal de 1969, comme il a été publié, continue avec la même philosophie de la peine du statut de 1940 encore en vigueur, il n'y aura aucune possibilité réelle pour la solution du problème des prisons au Pays, dont l'échec est unanimement reconnu"

Le mois d'août 1972, deux autres motions étaient proclamées à ce même sujet par les membres de la Commission de Droit Pénal du PREMIER Congrès de DRoIt de SÃo Paulo et par les participants brésiliens au Premier Cours International de Criminologie de L'Institut Oscar Freire qui ont au lieu à São Paulo.

Cela suffit pour démontrer qu'il existe un désir commum de modifier le système des peines pour chercher des solutions pour l'angoissant problème des prision au Brésil.

\section{EXAMEN PAR RAPPORT AUX HOMICIDES ET AUX CRIMES CONTRE LE PATRIMOINE.}

Du point de vue de la criminalité traditionelle, l'accroissement des crimes d'homicide et des crimes contre le patrimoine, ces derniers accompagnés d'une violence croissant, est inquétant. La croissant évolution de cette violence est constatée par les statistiques le moins prétentieuses. Dans un article publié la revue Vida Forense de São Paulo, n. ${ }^{\circ} 28$, du mois de septembre dernier, le journaliste et professeur de droit $\mathrm{Mr}$. Teofilo Cavalcanti Filho a discouru sur "La crise de la criminalité" Parmi d'autres observations, l' auteur a dit: "Ce n'est plus possible de ne pas reconnaitre que nous sommes en train de traverser une phase particulièrement grave et dangereuse à propos de la délinquance. Plus que jamais la délinquance a pris, parmi noux, des fonmes clairement agressives. Les attentats à la vie, accompagnés d'attentats à la propriété, deviennent routiniers dans les jornaux. Les délits contre la propriété accompagnés de meurtre sont si nombreaux qu' ils commencent à nous causer de l'alarme. Les délinquants, en se servant des moyens les plus avancés que la technique a engendrée ne réculent pas devant la force organisée de sûreté". 
Et l'auteur continue: "Én ce qui concerne l'homicide et le vol accompagné, évidemment les peines ne peuvent pas être considérées comme non satisfaisantes. L'homicide simple est puni par notre Droit avec des peines se prolongeant de 6 à 20 ans de réclusion.

Le vol accompagné de meurtre se place entre des limites encore plus hautes: de 15 a 30 ans de réclusion". Par conséquent il déclare qu'il n'est pas correct d'affirmer que l'accroissement de la criminalité se doit à l'insuffisance du quantum des peines, comme il a semblé correct à ceux qui ont pensé au rétablissement de la peine de mort.

Le phénomène est universel et préoccupe également tous les pays. L'explication causale qu'on essaye de donner ne peut pas être unitaire. De multiples facteurs sont à la génèse de ces délits réclamant une observation et une analyse soigneuse. Il est important de noter la présence continuelle des mineurs irresponsables parmi les auteurs de ces attentats contre la vie et la propriété. Il ne s'agit plus d'une simple délinquance juvénile, qu'on la considère des actes de vandalisme ou de contestation, mais des manifestations dangereuses de criminalité explosive, outre passant les limites fixées par l'imagination la plus outrée.

Devant une telle réalité, qu'on ne peut ni diminuer ni cacher, il faut réagir constructivement, en planifiant les moyens de combat capables de, du moins, nous acheminer vers une solution satisfaisante de ce problème.

La criminologie du passé ne suffit pas à donner au Droit pénal et à la Politique criminelle des règles à suivre. Il faut créer une nouvelle des règles à suivre. Il faut créer une nouvelle mentalité pour l'étude et l'enseignement de cette science en la faisant objet d'étude des Facultés de Droit, en lui donnant la charge de, aidée par d'autres études, montrer le chemin correct de la recherche. Et il devient impérative la création par le Ministère de la Justice d'un organe officiel qui, suivant les modèles la LEAA nord-américaine, centralise les études, les plans et les recherches criminologiques.

\section{SYNTHÈSE FINALE.}

De tout ce qu'on vient de dire on conclut qu'on a besoin du rapprochement de la Criminologie et du Droit pénal, séparés dès qu'on a jugé 
incovenable pour la dogmatique pure la présence d'élements meta-juridiques. La distinction entre sciences naturelles et sciences culturelles n'intervient plus d'une façon décisive dans leurs relations. Sans l'aide de la Criminologie, ou ne sortira qu' avec peine de l'impasse où nous trouvons. Cepedant, toute seule, la Criminologie ne peut resoudre les graves problèmes qu'on y a examinés.

La recherche des solutions substitutives est vaine. Elles ne serviront qu'à retarder le dénouement qu' on prévoit au sommet de la crise par laquelle nous sommes enveloppés. Quoique nous vivions dans un pays qui ne fait que les premiers pas sûrs vers le developpement, il faut disposer pour l'avenir, parce que nous avons de sûrs indices que le Brésil sera la nouvelle scène choisie par la criminalité organisée.

Ecoutons ce conseil sérieux donné par Mr. Eduardo NovoA MoNREAL: "Si nous voulons que le droit continue comme une règle ferme de conduite dans les sociétés modernes, capable d'assurer à tous les hommes la libre possibilité d'un developpement intégral, nous autres les juristes devrons nous convaincre que nous n'avons pas d'autre solution, sinon moderniser le droit et le rebâtir sur des bases lui permettant de s'adapter, avec la flexibilité nécessaire, aux changements nouveaux et certainement plus accélérés que nous devrons voir dans l'avenir" 4.

4. EDUARDO NOVOA MONREAL, art. cit., p. 10. 